\title{
Comprehensive Analysis of Etiology on the Prognosis of Urethral Strictures
}

\author{
Rajkumar Mathur, Gaurav Aggarwal, Bhaskar Satsangi, Fareed Khan, Sudarshan Odiya
}

Department of Surgery, M.G.M Medical College \& M.Y.H group of Hospitals, Indore, Madhya Pradesh, India

\begin{abstract}
Introduction: Urethral strictures remain a reconstructive dilemma, due to high incidence of recurrence and less than satisfactory outcomes. Even experienced surgeons following strict surgical principles have not achieved optimal results, leading us to think whether

the etiology of strictures dictate the outcome. We evaluated this "cause-effect" relationship highlighting the significance of the etiology on the overall prognosis of urethral strictures.

Materials and Methods: A total of 302 males with urethral strictures were assessed (both retrospectively and prospectively) over a period of ten years. The preoperative evaluation was performed by retrograde urethrogram, urethrosonogram, and uroflowmetry and categorized, based on etiology: a) as post traumatic, b) post infective, c) iatrogenic or d) unknown. Traumatic strictures were subjected to pelvic X-ray and sub-categorized into grades A, B and C, following the TILE classification. Patients were operated; with tunica albuginea urethroplasty for anterior strictures and $\mathrm{U}$ shape prostato-bulbar anastomosis for posterior strictures.

Results: Traumatic strictures accounted for $54 \%$ of cases. 127 of the 302 patients were treated using Tunica Albuginea Urethroplasty, while U shaped Prostatobulbar Anastomosis was performed on others. Post traumatic strictures had best outcome whereas post infective strictures had the worse outcome. Among strictures following pelvic fractures, TILE grades A and B had a better post operative course as compared to TILE C. Overall complication rate was $13.24 \%$.

Conclusion: Our study demonstrated that etiology of urethral strictures may play a vital role for the overall prognosis of urethral strictures.
\end{abstract}

Key words: Prognosis; urethral stricture; treatment outcome; etiology.

Int Braz J Urol. 2011; 37: 362-370

\section{INTRODUCTION}

Urethral Stricture Disease encompasses a spectrum of divergent ailments that cause obliteration of the urethral lumen and slowing or blocking urinary flow, secondary to fibrosis and healing of the urethral mucosa and surrounding tissues.

Urethral strictures have been a reconstructive dilemma for many years due to the high incidence of recurrence and less than satisfactory outcomes. A thorough preoperative evaluation, appropriate surgical planning, and adherence to basic surgical principles, even in the hands of the most experienced surgeon, have failed to achieve the desired results.

This has shifted the entire onus on the impact of a yet unexplored entity- the etiology.

Moreover, a stricture is a stricture, be it anterior or posterior and any etiology responsible for its causation, may lead to either an anterior or posterior variety of stricture. 
Strictures are a common iatrogenic sequel to urethral instrumentation and catheterization. Infective strictures have taken a back seat when compared with the rate of pelvic trauma and urethral manipulation, as well as with the advent of newer antibiotics and increasing public awareness as regards to sexually transmitted diseases (1).

Despite advancements in the field of reconstructive urology, there is no consensus as the best mode of treating this complex entity, in order to optimize the results.

We evaluated the "cause- effect" relationship between etiology of urethral stricture and outcome post-urethroplasty.

\section{MATERIALS AND METHODS}

This study included 302 male patients presenting with signs and symptoms of urethral stricture, for the first time, over a period of ten years, both retrospectively as well as prospectively, from 1999 to 2009 [RKM, GA, BS, FK, SO]. Congenital strictures, as well as, patients managed without surgical reconstruction were excluded from the study.

A detailed preoperative assessment included careful history-taking and physical examination; followed by stricture evaluation via retrograde urethrogram, urethrosonogram, and uroflowmetry. Patients were categorized, based on their stricture etiologies, as post traumatic, post infective, iatrogenic (post catheterization and post instrumentation), or unknown.

Patients with traumatic urethral strictures were then subjected to pelvic X-rays in both anteroposterior and lateral views, to identify the presence, as well as, type of pelvic fracture, and further subcategorize them into grades A, B and C, of the TILE classification (Table-1).

Irrespective of the etiology, if any patient had chronic retention of urine or complete outflow obstruction not amenable to urethral drainage, a urinary diversion in the form of suprapubic cystostomy was done.

A minimum wait of 6 weeks was allowed before urethroplasty to patients that required suprapubic cystostomy. Tunica albuginea urethroplasty (TAU) was the procedure of choice for anterior urethral strictures and U shaped prostato-bulbar anastomosis (USPBA) for posterior urethral strictures, in order to minimize the influence of the surgical procedure on the outcome $(2,3)$. No modifications were performed as regards to the surgical techniques, over the course of the study.

Post urethroplasty, a silicon catheter was kept for three weeks in case of simple strictures and for six weeks in complex strictures. Complex cases included the combined strictures of both the anterior and posterior urethra and the posterior urethral strictures with false passages resulting from multiple attempts of urethral instrumentation before the patients were referred to our center.

Table 1 - TILE Classification of Pelvic Fractures.

\begin{tabular}{llll}
\hline Tile Grade & Fracture Type & Fractures Included & Fracture Management \\
& & & \\
\hline \multirow{2}{*}{ Stable, } & Minimally displaced. & Avulsion pelvis, & Conservative. \\
& & Iliac wing, & Isolated pubic rami, \\
& Rotationally unstable, & Ondisplaced acetabular. & \\
& Vertically stable. & Ipsilateral pubic rami with posterior & Conservative, occasionally require \\
& & complex injury, & \\
& & Bucket handle. & \\
& & Unilateral pelvic, & Always require traction and/ \\
& Rotationally unstable, & Bilateral pelvic [anterior \& posterior], & or fixation. \\
& Vertically unstable. & Acetabular with unilateral or & \\
& & bilateral pelvic. & \\
\hline
\end{tabular}


Patients were scheduled for an initial postoperative assessment at the end of 3 months, with contrast urethrogram, urethrosonogram, and uroflometry. Post operative results were assessed by comparing pre and postoperative investigations and patient satisfaction (Table-2) and similarly at subsequent follow ups, at 6,12 and 24 months to evaluate the long term results and complications.

\section{RESULTS}

The results are exemplified both descriptively and statistically. Most of our patients were young with a mean age of 35.5 years old (range: $15-65$ years).
Prostatobulbar Anastomosis was performed in 175 patients (48\%).

Results were quantified at regular intervals on the basis of the criteria enlisted in Table-1, evaluating patient's satisfaction.

Loss of follow-up is a well recognized challenge in any study but we collected data in $82 \%$ of our patients for two years.

A comparison of the etiology regarding to success rates depicted that post traumatic strictures had the best overall outcome. Whereas, post infective variety had the poorest results (Figure-1).

Among the strictures following pelvic fractures, those in TILE grades A and B had a better

Table 2 - Postoperative Result Assessment Criteria.

\begin{tabular}{llll}
\hline & GOOD & FAIR & POOR \\
\hline Retrograde Urethrogram & Good caliber. & $\begin{array}{l}\text { Partial narrowing } \\
\text { at stricture site. }\end{array}$ & Persistent stricture. \\
Urethrosonogram & Patent and distensible & $\begin{array}{l}\text { Patent lumen with } \\
\text { decreased distensibility }\end{array}$ & Stricture present. \\
Uroflowmetry & Qmax $>20 \mathrm{~mL} / \mathrm{sec}$ & Qmax: $15-20 \mathrm{~mL} / \mathrm{sec}$ & Qmax $<15 \mathrm{~mL} / \mathrm{sec}$ \\
Patient Satisfaction & Satisfactory voiding, & Satisfactory voiding & Not satisfied, not voiding or \\
& No instrumentation & but required & voiding with thin stream, \\
& needed. & dilatations. & need multiple dilatations or \\
& & & repeat surgery. \\
\hline
\end{tabular}

Traumatic strictures were the most common etiology found, accounting for $54 \%$ of all cases (Table-3). Although the most common stricture location was the membranous urethra (35\%), the bulbar $(30 \%)$ and penile $(27.5 \%)$ urethra were equally affected and $7.5 \%$ patients had multiple urethral strictures.

Preoperative maximum flow rate (Qmax) analysis showed a range of 3.0 to $12.5 \mathrm{~mL} / \mathrm{s}$, the mean flow rate was $7.6 \mathrm{~mL} / \mathrm{s}$, which improved to 32 $\mathrm{mL} / \mathrm{s}$ post operatively, ranging from $18 \mathrm{~mL} / \mathrm{s}$ to 46 $\mathrm{mL} / \mathrm{s}$.

Average stricture length measured intraoperatively was $3.2 \mathrm{~cm}$, ranging from 2.5 to 12.5 $\mathrm{cm}$. A total of 127 patients $(42 \%)$ were treated using Tunica Albuginea Urethroplasty, while U shape post operative course as compared to TILE C variety (Figure-2).

Overall complication rate in our study was $13.24 \%$ (40 patients). Re-stenosis occurred in 4.6\% patients second only to post operative infection $(6.9 \%)$. Analyses of complications based on the primary cause are shown in Table-4.

\section{DISCUSSION}

Various studies have been done to identify the major etiologies for stricture formation, notwithstanding their actual impact on the long term outcome post surgical intervention.

In 1981, a review of 20 articles between 1961 and 1981, regarding the etiology of strictures, 
Table 3 - Various etiologies encountered.

\begin{tabular}{lccc}
\hline S. No. & Etiology & \multicolumn{2}{c}{ Number of cas es } \\
\cline { 3 - 4 } & & Number & \% of cases \\
\hline 1. & Pelvic trauma & 163 & $54 \%$ \\
2. & Post instrumentation & 17 & $5.6 \%$ \\
3. & Post catheterization & 64 & $21.1 \%$ \\
4. & Infection & 46 & $15.2 \%$ \\
5. & Unknown & 12 & $3.9 \%$ \\
\hline
\end{tabular}

was published, with urethritis being the most common cause at that time, in $40 \%$ of the cases (4). Other major series in the developed world, also report a $32 \%$ to $79 \%$ rate of iatrogenic causes $(5,6)$. Jordan and Schlossberg, in 2002, suggested that most strictures in the recent era are a result of external trauma (7). Our study also observed such transition to the etiology of strictures, with $54 \%$ incidence of urethral strictures occurring post trauma.

Traumatic strictures most frequently involve the posterior urethra. Pelvic fracture causing disruption at the bulbo-membranous junction. Studies have reported an incidence of such strictures up to $31 \%$, in countries with poor road conditions, as well as, inadequate traffic regulations (8). Casselman and Schillinger hypothesized that the mechanism of injury to the urethra in major pelvic fracture cases involves compression of the pelvic ring laterally, resulting in an increase in the antero-posterior diameter, leading to superior bladder displacement and consequent urethral stretching and avulsion (9). In our study, $54 \%$ of strictures encountered were of the traumatic etiology, with $84 \%$ of these patients having radiological evidence of pelvic fractures. Various types of pelvic fractures were seen, majority of injuries classified as grades A and B (83.2\%) of the TILE classification system (10), with involvement of the inferior pubic rami being the most common type.

The TILE classification of pelvic fractures is based on progressive instability of the pelvis (10). Type A fractures are stable with respect to rotation and vertical displacement, whereas type B fractures are rotationally unstable and vertically stable. Type C fractures are both vertically and rotationally unstable.
Classifying by level of instability is valuable in predicting morbidity and mortality, as the pattern of urethral injury can be predicted on the basis of the type of pelvic fracture. The highest risk of urethral injury is found in a straddle fracture combined with diastasis of the sacroiliac joint (11). For every $1 \mathrm{~mm}$ increase of the pubic symphyseal diastasis, or displacement of the infero-medial pubic bone fracture fragments, the risk of urethral injury increases by $10 \%$ (12).

Post traumatic strictures tend to be short, occurring exclusively in the bulbar (5) and membranous urethra $87.73 \%$ of traumatic strictures in our study were seen to involve these sites, and $85 \%$ of these were less than $4 \mathrm{~cm}$ in length.

Management of post traumatic strictures usually involves preliminary diversion by suprapubic cystostomy, followed by definitive repair several weeks later. The delayed urethroplasty after 3 months after initial trauma reduces complications of impotence and incontinence. In addition, the major advantage of delayed urethral reconstruction is that it can be done under controlled conditions when the patient has recovered from major associated injuries (13).

In our study, 6 weeks post suprapubic cystostomy, these patients were taken up for surgery; with tunica albuginea urethroplasty (TAU) being our procedure of choice for anterior urethral strictures and U shaped prostato-bulbar anastomosis (USPBA) for posterior urethral strictures $(2,3)$.

Postoperatively, periodic evaluation revealed a decline in the success rate over a period of time as depicted in our previous studies $(2,3)$. A similar trend was evident in post traumatic strictures in the present study. Furthermore, the results were much better in 


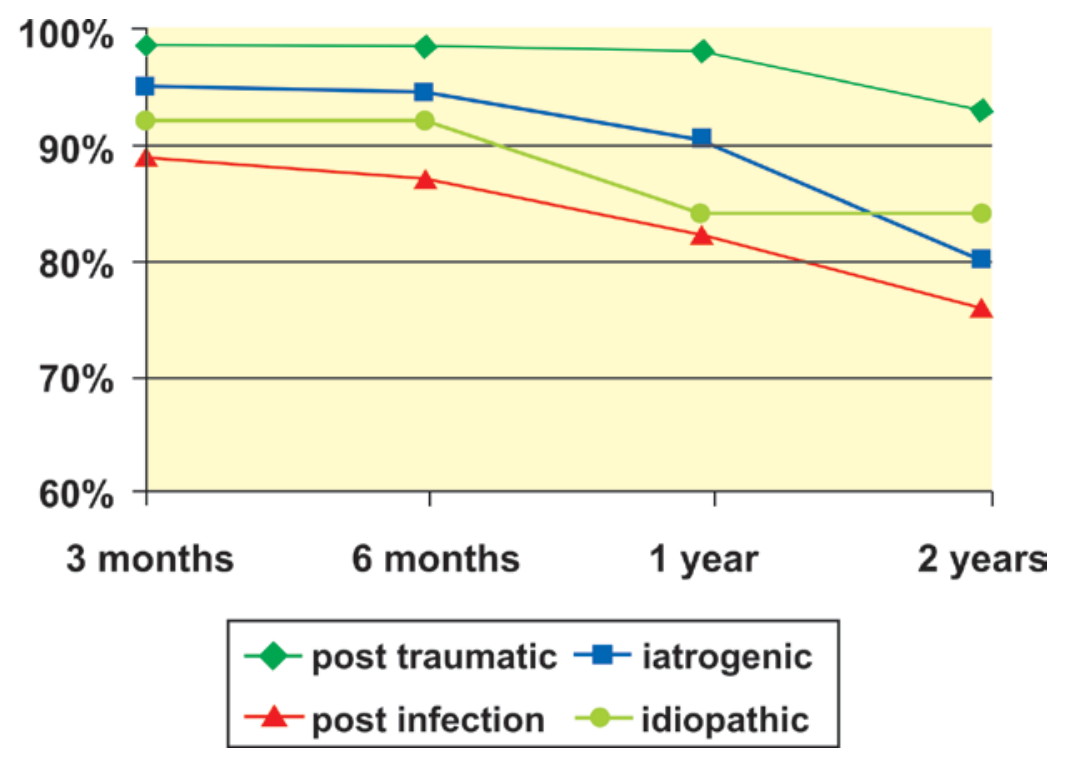

Figure 1 - Etiology and success rates.

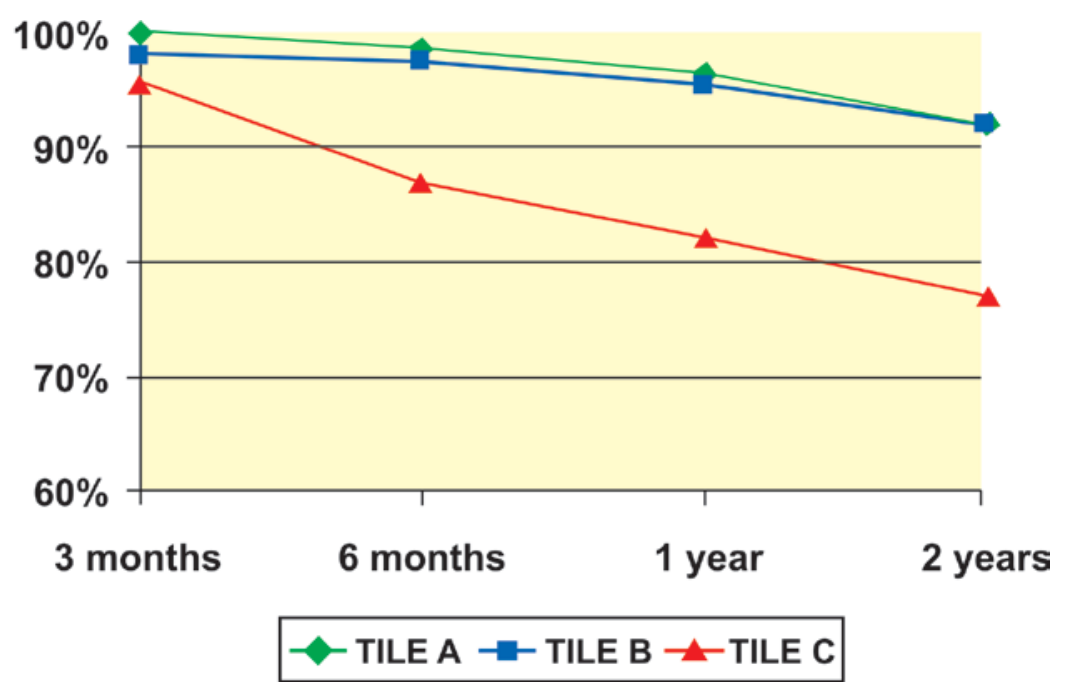

Figure 2 - Success rates based on TILE grading.

patients falling in TILE category A, with $92 \%$ success rate at 2 years follow-up, when compared with $91.5 \%$ for TILE grade B and 77\% in cases of TILE C, at the same time.

Numerous studies support the occurrence of complications after urethral injury following a pelvic fracture, due to the injury itself rather than the surgical technique (14-16). We encountered complications in 26 patients with post traumatic strictures: infection, re-stenosis, impotence, urinary incontinence, urethracutaneous fistulae, wound dehiscence and ejaculatory disturbances, with rates acceptable in comparison to other studies $(17,18)$.

Iatrogenic urethral injuries producing urethral strictures result from urethral instrumentation, urethral catheterization, and rarely following abdominoperineal resection for carcinoma of the rectum (19). Prolonged catheterization and transurethral surgery 
Table 4 - Complication rates based on stricture etiologies.

\begin{tabular}{|c|c|c|c|c|c|c|c|c|}
\hline S.No. & Complication & Infection & Restenosis & Impotence & Incontinence & UCF & $\begin{array}{c}\text { Wound } \\
\text { Dehiscence }\end{array}$ & $\begin{array}{c}\text { Ejaculatory } \\
\text { problems }\end{array}$ \\
\hline & Etiology & {$[6.9 \%]$} & {$[4.6 \%]$} & {$[1.6 \%]$} & {$[2.6 \%]$} & {$[1 \%]$} & {$[2.6 \%]$} & [1\%] \\
\hline 1. & Pelvic trauma & $6[3.6 \%]$ & $6[3.6 \%]$ & $3[1.8 \%]$ & $5[3 \%]$ & $\begin{array}{c}1 \\
{[0.6 \%]}\end{array}$ & $4[2.4 \%]$ & $1[0.6 \%]$ \\
\hline 2. & Iatrogenic & $7[8.6 \%]$ & $5[6.1 \%]$ & $1[1.2 \%]$ & $2[2.4 \%]$ & $\begin{array}{c}1 \\
{[1.2 \%]}\end{array}$ & $1[1.2 \%]$ & $2[2.4 \%]$ \\
\hline 3. & Infection & $\begin{array}{c}7 \\
{[15.2 \%]}\end{array}$ & 3 [6.5\%] & $1[2.1 \%]$ & $1[2.1 \%]$ & $\begin{array}{c}1 \\
2.1 \%]\end{array}$ & $2[4.3 \%]$ & 0 \\
\hline 4. & Unknown & $1[8.3 \%]$ & 0 & 0 & 0 & 0 & $1[8.3 \%]$ & 0 \\
\hline
\end{tabular}

are the main reasons for the ischemic urethral insult. A rate of $32 \%$ to $79 \%$ of iatrogenic causes has been reported in different studies $(5,6)$. We recorded a $26.8 \%$ incidence of iatrogenic urethral strictures.

Urethral stricture is a major late complication of TUR $(2.2 \%$ to $9.8 \%$ of cases $)(20,21)$, as well as, radical (8.4\%) (22) and simple (1.9\%) (23) prostatectomy. Numerous causes of stricture formation postTUR have been suggested, such as improper traumatic insertion of the resectoscope with perforation of the bulbous urethra and instrument friction at the penoscrotal angle, eventually exacerbated by the narrow urethral caliber as well as monopolar current "leak" due to insufficient resectoscope isolation (20). Moreover, patients undergoing such urological procedures are also catheterized in the post operative period. The exact cause, however, remains undetermined to date.

Prolonged catheterization, as in multiple trauma or burn patients, leads to urethral inflammation and ischemia, and ultimately stricture formation (5). Moreover, improper urethral catheter insertion is a preventable source of injury, with a recent report suggesting that 3.2 urethral injuries per 1000 inpatients occurred due to improper urethral catheterization (24).

Post instrumentation strictures are usually short and well defined, commonly located in the bulbomembranous urethra, whereas those occurring post catheterization tend to be long and irregular, and more common at the penoscrotal junction (19), correlating well with our findings. A total of $60 \%$ of post instrumentation strictures encountered by us were at the membranous and bulbar urethra, while $48 \%$ of post catheterization strictures were located at the penoscrotal junction.
Iatrogenic strictures frequently require elaborate reconstructive procedures (25). Repeated urethral dilatation exacerbates short strictures, making them longer (26). The reconstructive urologist must consider the etiology, as well as, the prior treatments when planning whether to excise or graft the stenotic urethral segment.

Post operative short term follow-up results were encouraging for post traumatic strictures but the long term results were not so good.

Infection and re-stenosis were the most frequent complications encountered $\mathrm{s}$, more so in the case of post catheterization strictures, whereas impotence, incontinence, urethra-cutaneous fistulae, wound dehiscence and ejaculatory disturbances were also seen.

Nevertheless, adequate and strict indications for urinary catheterization, skilled urethral catheter insertion and consideration of suprapubic catheter placement whenever prolonged catheterization is anticipated, should decrease the incidence of these iatrogenic strictures, which often tend to be multifocal or panurethral (27), and thus more difficult to treat.

Inflammatory strictures may be seen with gonorrheal infection, nonspecific infections by chlamydia and ureaplasma urealyticum, as well as, lichen sclerosus, usually beginning with inflammation of glans and inevitably leading to meatal stenosis. Progression of stricture may involve the anterior and posterior urethra (28).

We encountered 46 patients (15.2\%) with post infective strictures, with $66 \%$ of these located in the penile and bulbar urethra. Escherichia Coli was the most common isolate from the urine culture in these patients, suggesting that urethral obstruction 
may induce organisms which are cleared rapidly from the normal urinary tract, to cause bacteruria, bacteremia and even pyelonephritis (29).

Post urethroplasty evaluation at 6 months showed $86.95 \%$ success rate, which reduced to $75.67 \%$ at 2 years. A high rate of infection (15.2\%) and re-stenosis $(6.5 \%)$ was seen in the follow-up of inflammatory strictures, correlating with other similar studies. None of the patients, however, faced any ejaculatory problems. Post infective strictures was the worst in terms of overall prognosis.

Idiopathic strictures or strictures without any apparent reason have been observed in different studies $(5,7,29)$. $3 \cdot 9 \%$ of our patients fell into this category.

Although several theories have formulated, its mechanism remains unknown. But it may occur as a delayed manifestation of unrecognized childhood trauma, even presenting as long as 18 years after perineal trauma (1). Many idiopathic strictures may develop as a consequence of inadequate canalization, when the part of the urethra derived from the urogenital sinus joins the part derived from the urogenital folds, becoming symptomatic with growth (30). In elderly men, another explanation postulated is that idiopathic strictures are mainly ischemic in origin (29).

These strictures are significantly more prevalent in the bulbar area and more common in the younger age group (31). A total of $59 \%$ of idiopathic strictures in our study involved the bulbar urethra.

Our study demonstrates that post traumatic urethral strictures, secondary to pelvic fractures of the TILE category A, are shorter; mainly involve the bulbar and membranous urethra, post surgical intervention have the best long term outcome, as well as, the lowest complication rate, and the best prognosis.

\section{CONCLUSIONS}

Post urethroplasty success has been attributed to sevral factors,i.e.; location and length of the stricture, degree of spongiofibrosis, the choice of surgical procedure and surgical expertise. However, the impact of etiology regarding to outcome has remained unexplored. Our study demonstrated that the etiology may playsa vital role to the overall prognosis of urethral strictures treatment.

\section{CONFLICT OF INTEREST}

None declared.

\section{REFERENCES}

1. Baskin LS, McAninch JW: Childhood urethral injuries: perspectives on outcome and treatment. Br J Urol. 1993; 72: 241-6.

2. Mathur RK, Himanshu A, Sudarshan O: Technique of anterior urethra urethroplasty using tunica albuginea of corpora cavernosa. Int J Urol. 2007; 14: 209-13.

3. Mathur RK, Aggarwal H, Odiya S, Lubana PS: Ushaped prostatobulbar anastomosis for urethral injury after pelvic trauma. ANZ J Surg. 2008; 78: 605-9.

4. De Sy WA, Oosterlinck W, Verbaeys A: Le traitement du retrecissement de 1 uretre masculine. Acta Urol Belg. 1981; 49: 101-2.

5. Fenton AS, Morey AF, Aviles R, Garcia CR: Anterior urethral strictures: etiology and characteristics. Urology. 2005; 65: 1055-8.

6. Barbagli G, Guazzoni G, Lazzeri M: One-stage bulbar urethroplasty: retrospective analysis of the results in 375 patients. Eur Urol. 2008; 53: 828-33.

7. Jordan GH, Scholssberg SM: Surgery of the penis and urethra, (In) Walsh PC, Retik AB, Vaughan ED Jr, et al. (ed.), Campbell's Urology, 8th ed. WB Saunders, Philadelphia, 2002; pp. 3886-952.

8. Tazi K, Nouri M, Med Moudouni S, Koutani A, Benatyaa A, Hachimi M, et al.: Traitment des stenoses inflammatoires de 1 uretre par uretrotomie endoscopique. Ann Urol 2000; 34: 184-8.

9. Casselman RC, Schillinger JF: Fractured pelvis with avulsion of the female urethra. J Urol. 1977; 117: 3856.

10. Tile M: Pelvic ring fractures: should they be fixed? J Bone Joint Surg Br. 1988; 70: 1-12.

11. Mouraviev VB, Santucci RA: Cadaveric anatomy of pelvic fracture urethral distraction injury: most injuries are distal to the external urinary sphincter. J Urol. 2005; 173: 869-72.

12. Basta AM, Blackmore CC, Wessells H: Predicting urethral injury from pelvic fracture patterns in male patients with blunt trauma. J Urol. 2007; 177: 571-5.

13. Koraitim MM: Pelvic fracture urethral injuries: the unresolved controversy. J Urol. 1999; 161: 1433-41.

14. Kotkin L, Koch MO: Impotence and incontinence after immediate realignment of posterior urethral trauma: re- 
sult of injury or management? J Urol. 1996; 155: 16003.

15. Shenfeld OZ, Kiselgorf D, Gofrit ON, Verstandig AG, Landau EH, Pode D, et al.: The incidence and causes of erectile dysfunction after pelvic fractures associated with posterior urethral disruption. J Urol. 2003; 169: 2173-6.

16. Koraitim MM: On the art of anastomotic posterior urethroplasty: a 27-year experience. J Urol. 2005; 173: 135-9.

17. Santucci RA, Mario LA, McAninch JW: Anastomotic urethroplasty for bulbar urethral stricture: analysis of 168 patients. J Urol. 2002; 167: 1715-9.

18. Elliott SP, Metro MJ, McAninch JW: Long-term followup of the ventrally placed buccal mucosa onlay graft in bulbar urethral reconstruction. J Urol. 2003; 169: 1754-7.

19. McCallum RW, Rogers JM, Alexander MW: The radiologic assessment of iatrogenic urethral injury. J Can Assoc Radiol. 1985; 36: 122-6.

20. Rassweiler J, Teber D, Kuntz R, Hofmann R: Complications of transurethral resection of the prostate (TURP)--incidence, management, and prevention. Eur Urol. 2006; 50: 969-79; discussion 980.

21. Lumen N, Oosterlinck W: Challenging non-traumatic posterior urethral strictures treated with urethroplasty: a preliminary report. Int Braz J Urol. 2009; 35: 442-9.

22. Elliott SP, Meng MV, Elkin EP, McAninch JW, Duchane J, Carroll PR, et al.: Incidence of urethral stricture after primary treatment for prostate cancer: data From CaPSURE. J Urol. 2007; 178: 529-34; discussion 534.

23. Varkarakis I, Kyriakakis Z, Delis A, Protogerou V, Deliveliotis C: Long-term results of open transvesical prostatectomy from a contemporary series of patients. Urology. 2004; 64: 306-10.

24. Kashefi C, Messer K, Barden R, Sexton C, Parsons JK: Incidence and prevention of iatrogenic urethral injuries. J Urol. 2008; 179: 2254-7; discussion 2257-8.

25. Wessells H, Morey AF, McAninch JW: Single stage reconstruction of complex anterior urethral strictures: combined tissue transfer techniques. J Urol. 1997; 157: 1271-4.

26. Park S, McAninch JW: Straddle injuries to the bulbar urethra: management and outcomes in 78 patients. $\mathrm{J}$ Urol. 2004; 171: 722-5.

27. Ruutu M, Alfthan O, Talja M, Andersson LC: Cytotoxicity of latex urinary catheters. Br J Urol. 1985; 57: 827.

28. Venn SN, Mundy AR: Urethroplasty for balanitis xerotica obliterans. Br J Urol. 1998; 81: 735-7.

29. Depasquale I, Park AJ, Bracka A: The treatment of bal- anitis xerotica obliterans. BJU Int. 2000; 86: 459-65.

30. Andrich DE, Mundy AR: What is the best technique for urethroplasty? Eur Urol. 2008; 54: 1031-41.

31. Lumen N, Hoebeke P, Willemsen P, De Troyer B, Pieters R, Oosterlinck W: Etiology of urethral stricture disease in the 21st century. J Urol. 2009; 182: 983-7.

Accepted after revision:

November 16, 2010

\author{
Correspondence address: \\ Dr. Rajkumar Mathur \\ Head, Department of Surgery \\ M.Y. Hospital and M.G.M Medical College \\ Indore, Madhya Pradesh, 452001, India \\ Fax: + 967098 2604-0088 \\ E mail:rkmmyh70@yahoo.com
}

\section{EDITORIAL COMMENT}

Mathur and co-workers present a very extensive series on urethral stricture disease and its management, as well as, prognostic factors. In their analysis they found that more than $54 \%$ of the strictures were posttraumatic. Moreover they are able to even correlate pelvic fracture patterns with individual outcome. Overall complication rate with less than $15 \%$ is remarkably low in such a complex cohort of patients. In addition to the well-known technique of prostatobulbar anastomosis, the authors employed their own technique of for anterior reconstruction. The so-called tunica albuginea urethroplasty offers the advantage of locally available substitute tissue even in case of oral disease precluding the use of buccal mucosa (1). As such we do require alternative graft sources in patients with oral disease allowing for comparable complication rates (2). Genital skin remains such an alternative donor site being important for the reconstructive armamentarium. The authors clearly demonstrate that post-inflammatory strictures yielded a poor outcome as compared to the posttraumatic ones. In fact this observation is most likely due to underestimating the de- 
gree and extension of spongiofibrosis. Ultrasound has been shown to be a valuable tool for this task before urethroplasty (3).

Nevertheless, Mathur et al remain to be congratulated for the comprehensive analysis of stricture etiology, pelvic fracture pattern and individual surgical outcome. Being aware of the relation between these features and surgical success allows us for a more accurate preoperative counseling in our patients.

\section{REFERENCES}

1. Mathur RK, Himanshu A, Sudarshan O: Technique of anterior urethra urethroplasty using tunica albuginea of corpora cavernosa. Int J Urol. 2007; 14: 209-13.

2. Schwentner C, Seibold J, Colleselli D, Alloussi SH, Schilling D, Sievert KD, et al.: Dorsal onlay skin graft urethroplasty in patients older than 65 years. Urology. 2010; 76: 465-70.

3. Mitterberger M, Christian G, Pinggera GM, Bartsch G, Strasser H, Pallwein L, et al.: Gray scale and color Doppler sonography with extended field of view technique for the diagnostic evaluation of anterior urethral strictures. J Urol. 2007; 177: 992-6; discussion 997.

\author{
Dr. C. Schwentner \\ Department of Urology \\ Eberhard-Karls-University Tuebingen \\ Tuebingen, Germany \\ E-mail:christian.schwentner@i-med.ac.at
}

\section{EDITORIAL COMMENT}

This paper evaluated 302 patients with urethral strictures discussing its causes, the importance on prognosis and surgical outcomes according to treatment options. The lack of prospective studies in this segment of reconstructive urology represents a barrier on defining the best treatment modality for a very common subject among us urologists.

A recent publication from this group has shown the importance of urethral stricture related to pelvic fractures after a representative study on patients presenting pelvic trauma (1). Focus on the detailed diagnosis of the fractures and their co-morbidity was given, being possibly the reason for a research extension directed to etiology and surgical outcomes. This relation was also seen in the works of Bjurlin (2) and Pavelka (3) reinforcing the relevance of these findings.

Tunica albuginea urethroplasty and $\mathrm{U}$ shaped prostato-bulbar anastomosis for urethral strictures are extremely challenging surgical exercises even in the best of hands. Excellent long-term results can be expected especially when adequate excision of fibrotic tissue as well as wide-caliber, tension-free, bulboprostatic anastomosis is done. The outcomes reported in this study do corroborate with this affirmation, evidencing the experience of the authors.

Although a descriptive etiology related study is proposed, potential limitations of the paper are seen in the study design and number of surgeons who took part in this 10 year follow-up, as well as, incomplete post urethroplasty data. These results are extreme valuable and we encourage the authors to deepen their interest in this direction.

\section{REFERENCES}

1. Mathur R, Aggarwal G, Satsangi B, Khan F, Odiya S: Prognosis of urethral strictures following pelvic fracture urethral distraction defects-A single centre study. Int J Surg. 2010; 29. [Epub ahead of print]

2. Bjurlin MA, Fantus RJ, Mellett MM, Goble SM: Genitourinary injuries in pelvic fracture morbidity and mortality using the National Trauma Data Bank. J Trauma. 2009; 67: 1033-9.

3. Pavelka T, Houcek P, Hora M, Hlavácová J, Linhart M: Urogenital trauma associated with pelvic ring fractures. Acta Chir Orthop Traumatol Cech. 2010; 77: 18-23

\author{
Dr. R. Rossi Neto \\ Urologische Universitätsklinik Essen \\ Hufelandstrasse 55 \\ 45122, Essen, Germany \\ E-mail: roberto.rossineto@uk-essen.de
}

